\title{
Aortic symmetry index: Initial validation of a novel preoperative predictor of recurrent aortic insufficiency after valve-sparing aortic root reconstruction
}

\author{
Antonino Di Franco, MD, ${ }^{a}$ Lisa Q. Rong, MD, ${ }^{b}$ Monica Munjal, MS, ${ }^{a}$ Jonathan W. Weinsaft, MD, ${ }^{c}$ \\ Jiwon Kim, MD, ${ }^{\mathrm{c}}$ Francesco Sturla, PhD, ${ }^{\mathrm{d}}$ Leonard N. Girardi, MD, ${ }^{\mathrm{a}}$ and Mario Gaudino, MD, ${ }^{\mathrm{a}}$ New York, \\ NY, and Milan, Italy
}
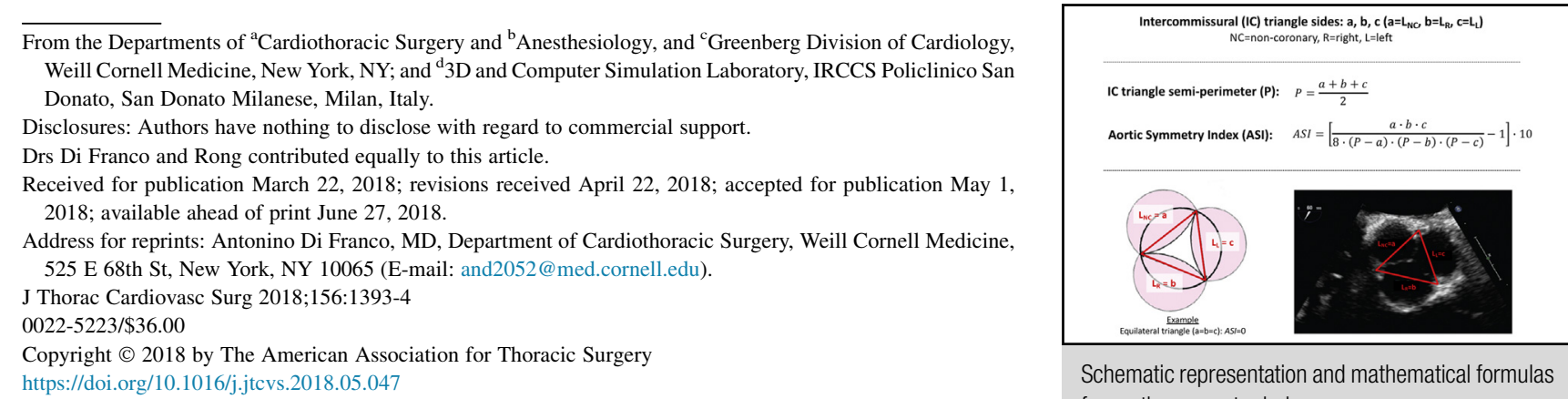

Schematic representation and mathematical formulas for aortic symmetry index.

Valve-sparing aortic root replacement (VSRR) has become increasingly favored relative to traditional valve replacement for patients with advanced aortic insufficiency (AI) because of its lower incidence of thromboembolic events, reduced risk of endocarditis, and avoidance of anticoagulation. ${ }^{1}$ Studies on determinants of recurrent AI after VSRR have primarily focused on postrepair measurements (eg, coaptation length and height). A preoperative predictor of successful VSRR, however, would hold the potential not only to influence the timing of surgical indication (similar to mitral valve surgery) but also to discriminate patients in whom VSRR is expected to be more easily feasible. This proof-of-concept study tested a novel preoperative echocardiographic predictor of recurrent AI among patients undergoing VSRR.

The study cohort was derived from patients with native trileaflet aortic valve (AV) undergoing VSRR at our institution (Weill Cornell Medicine, New York, NY) between 2006 and 2015. VSRR was performed with the classic David 1 technique, including the use of a straight Dacron polyester fabric tube (MAQUET Cardiovascular, LLC, Wayne, NJ). Preprocedural transesophageal echocardiography was used to quantify the degree of AV symmetry in the short-axis view: intercommissural distances at end diastole (as confirmed by electrocardiography) were measured to identify the "intercommissural triangle" connecting the 3 commissures. An "aortic symmetry index" (ASI) was defined according to the intercommissural triangle aspect ratio, ${ }^{2}$ expressed in terms of the lengths of the sides. ASI is equal to 0 for an equilateral triangle, whereas it increases progressively as the 3 sides of the triangle differ from each other (details and formulas in Figure 1). ${ }^{2}$ The goal was to test whether ASI predicted postoperative AI at follow-up, as assessed by

color-flow Doppler and graded according to current guidelines. ${ }^{3}$ For study purposes, recurrent advanced AI was defined as at least moderate to severe $(\geq 3+)$. Echocardiograms were interpreted by experienced investigators (A.D.F. and L.Q.R.). No patients underwent AV cusp repair. Data were analyzed with the SPSS statistics package (version 22; IBM, Armonk, NY). Continuous variables were summarized as mean $\pm \mathrm{SD}$; categorical variables were represented as percentages. The Student $t$ test was used to compare continuous variables. Development of postoperative AI and follow-up were analyzed with KaplanMeier estimates and comparison by log rank. Intraoperator and interoperator agreements for ASI were assessed with Bland-Altman plots. This study was approved by the institutional review board; informed consent was waived.

Complete transesophageal echocardiography data sets were available for 67 patients with native trileaflet $\mathrm{AV}$ ( $47.6 \pm 14.7$ years old, $78 \%$ male, $31 \%$ with connective tissue disorders). At a mean follow-up of $2.9 \pm 2.6$ years, all patients were alive; no patient underwent reoperation on the AV. Thirty patients had no AI at follow-up, 23 had grade 1 

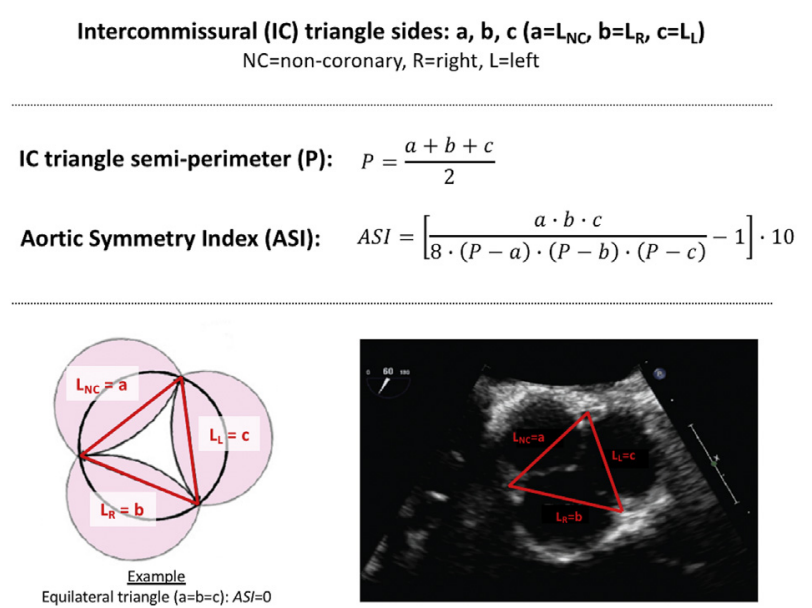

FIGURE 1. Schematic representation and mathematical formulas for aortic symmetry index $(A S I)$. IC, Intercommissural; $L_{N C}$, length of noncommissural side; $L_{R}$, length of right commissural side; $L_{L}$, length of left commissural side; $P$, semiperimeter of intercommissural triangle.

AI, 10 had grade $2 \mathrm{AI}, 3$ had grade $3 \mathrm{AI}$, and 1 had grade 4 AI. Follow-up was $100 \%$ complete.

ASI was quantifiable in $98.5 \%$ of cases and yielded excellent intraobserver and interobserver reproducibility $(\Delta,-0.06 \pm 0.10$ and $-0.04 \pm 0.07$, respectively). Magnitude of AV symmetry was significantly higher (ie, ASI closer to 0) in patients with no AI versus those with advanced AI at follow-up $(0.09 \pm 0.07$ vs $0.25 \pm 0.12$, respectively; $P<.001)$. Kaplan-Meier estimate compared ASI values of 0.10 or less and greater than 0.10 for AI grade $3+$ at follow-up, and log rank test revealed a $P$ value of .046 .

Success rate for VSRR is variable, and factors such as etiology of aortic valve pathology, valve and root morphology, and surgeon's experience all play a role. Surgical approaches for patients with AI can be conceivably changed or anticipated if there is a differential probability of successful VSRR, similar to what is suggested by guidelines for reparability of mitral valves in severe mitral regurgitation. ${ }^{4}$ Current predictors of recurrent AI after VSRR, however, have primarily focused on postrepair echocardiographic measurements. ${ }^{5}$
Our preliminary results suggest that ASI, a novel preoperative echocardiographic index, predicts postoperative success of VSRR. Of note, ASI is an easily obtainable echocardiographic index that could be used not only in a preoperative setting but also intraoperatively or at the end of the procedure to provide a quickly obtainable parameter to evaluate symmetry of the reimplanted valve. The concept is based on the intuitive idea that a more symmetric trileaflet $\mathrm{AV}$ is more likely to grant a successful repair than an asymmetric valve. We found that a lower ASI, indicating more symmetric AV (perfect symmetry being ASI $=0$ ) was significantly associated with no AI relative to those with AI of at least 3 at a mean follow-up of 2.9 years. Notably, ASI was quantifiable in almost all patients with trileaflet valves (98.5\% of the cases). Several limitations should be acknowledged, including the single-institution and retrospective design of the study, the limited sample size, the variability of the echocardiographer, and the image quality.

In conclusion, this validation study provides insights regarding a potentially important preoperative echocardiographic predictor of the successful VSRR. Further prospective studies in larger populations are warranted to further evaluate its utility.

\section{References}

1. Gaudino M, Lau C, Munjal M, Avgerinos D, Girardi LN. Contemporary outcomes of surgery for aortic root aneurysms: a propensity-matched comparison of valvesparing and composite valve graft replacement. J Thorac Cardiovasc Surg. 2015; 150:1120-9.e1.

2. Farrashkhalvat M, Miles JP. Unstructured grid generation. In: Basic Structured Grid Generation: With an Introduction to Unstructured Grid Generation. Oxford: Butterworth-Heinemann; 2003:190-226.

3. Zoghbi WA, Enriquez-Sarano M, Foster E, Grayburn PA, Kraft CD, Levine RA, et al; American Society of Echocardiography. Recommendations for evaluation of the severity of native valvular regurgitation with twodimensional and Doppler echocardiography. J Am Soc Echocardiogr. 2003; 16:777-802.

4. Nishimura RA, Otto CM, Bonow RO, Carabello BA, Erwin JP III, Guyton RA, et al; ACC/AHA Task Force Members. 2014 AHA/ACC guideline for the management of patients with valvular heart disease: a report of the American College of Cardiology/American Heart Association task force on practice guidelines. Circulation. 2014;129:e521-643. Erratum in: Circulation. 2014;129:e650.

5. le Polain de Waroux JB, Pouleur AC, Robert A, Pasquet A, Gerber BL, Noirhomme P, et al. Mechanisms of recurrent aortic regurgitation after aortic valve repair: predictive value of intraoperative transesophageal echocardiography. JACC Cardiovasc Imaging. 2009;2:931-9. 